\title{
Will the Systolic Blood Pressure Intervention Trial (SPRINT) change Treatment Targets in Hypertension?
}

Michael A Weber

\begin{abstract}
Most of the current guidelines recommend a target systolic blood pressure (SBP) of $<140 \mathrm{~mm} \mathrm{Hg}$ when treating hypertension. However, in the recent systolic blood pressure intervention trial (SPRINT) study which compared targets of 140 and $120 \mathrm{~mm} \mathrm{Hg}$ in patients at high cardiovascular risk and concluded that there were greater cardiovascular (CV) and mortality benefits at the lower target, including those patients aged above 75 years. The blood pressure (BP) measurement method in SPRINT was unconventional and the achieved SBP in the intensively treated patients corresponds to pressure in an orifice valve of about $130 \mathrm{~mm} \mathrm{Hg}$. Importantly, SPRINT excluded patients with diabetes or at low CV risk. These patients should be treated to $<140 \mathrm{~mm} \mathrm{Hg}$ since CV events may increase at more aggressive targets. In communities with limited resources it is often not possible to evaluate $\mathrm{CV}$ risk and $<140 \mathrm{~mm} \mathrm{Hg}$ should be the usual target; indeed, in Stage 1 hypertension, drug therapy can be delayed while lifestyle changes are tried.
\end{abstract}

Keywords: Cardiovascular risk, Hypertension guidelines, Systolic blood pressure, Systolic blood pressure intervention trial.

How to cite this article: Weber MA. Will the Systolic Blood Pressure Intervention Trial (SPRINT) change Treatment Targets in Hypertension? Hypertens J 2016;2(4):183-188.

Source of support: Nil

Conflict of interest: None

\section{INTRODUCTION}

The results of the systolic blood pressure intervention trial (SPRINT) have already impacted the field of hypertension. ${ }^{1}$ This trial compared intensive with standard blood pressure (BP) targets in hypertensive patients at high cardiovascular $(\mathrm{CV})$ risk and concluded that a target systolic $\mathrm{BP}$ of $<120 \mathrm{~mm} \mathrm{Hg}$ was superior to a target of $<140 \mathrm{~mm} \mathrm{Hg}$ in preventing major CV outcomes and mortality. We will discuss further details of SPRINT later in this brief commentary, but it was evident as soon as these results were announced and publicized in the lay media that the practice of clinical hypertension and the writing of formal hypertension practice guidelines would be influenced by this authoritative study.

\section{Professor}

Department of Cardiovascular Medicine, State University of New York, Downstate College of Medicine, Brooklyn, New York, USA

Corresponding Author: Michael A Weber, Professor, Department of Cardiovascular Medicine, State University of New York Downstate College of Medicine, Brooklyn, New York, USA, Phone: +7148157430, e-mail: michaelwebermd@cs.com

\section{BACKGROUND}

For several years, at least since the mid-1990s, most guidelines have recommended 140/90 $\mathrm{mm} \mathrm{Hg}$ as the threshold for diagnosing hypertension and that achieving BPs below this threshold should be the appropriate target of treatment., ${ }^{2,3}$ Although this widely used target was not based on any compelling randomized trial, the weight of evidence appeared to support a systolic target of around $140 \mathrm{~mm} \mathrm{Hg}$ for most adults.

One of the most influential studies in guiding this recommendation was the Systolic Hypertension in the Elderly Program in which active treatment, when compared with placebo, significantly reduced stroke and coronary events in patients aged 60 or older who had systolic BPs $\geq 160 \mathrm{~mm} \mathrm{Hg}$. ${ }^{4}$ In fact, the actively treated group finished with a mean systolic BP of $143 \mathrm{~mm} \mathrm{Hg}$, and the placebo group finished with $155 \mathrm{~mm} \mathrm{Hg}$. Since there appeared to be few if any safety issues with the active treatment, most experts felt comfortable recommending a target of $<140 \mathrm{~mm} \mathrm{Hg}$. As an interesting sidelight on this assumption, as recently as 2013, the so-called JNC 8 Report reinterpreted these data and recommended that, for patients aged 60 or more, a target of $<150 \mathrm{~mm} \mathrm{Hg}$ would be adequate. ${ }^{5}$ Not surprisingly, this recommendation was greeted with considerable questioning and uncertainty, but since the results of SPRINT have become available, it is now understood that the JNC 8 recommendation has doubtful validity.

A number of clinical trials, although not originally intended for this purpose, have supported the systolic target of $<140 \mathrm{~mm} \mathrm{Hg}$. Figure 1 represents an example of these data. In the valsartan antihypertensive long-term use evaluation trial, where investigators studying new $\mathrm{BP}$ medications were encouraged to achieve systolic BPs $<140 \mathrm{~mm} \mathrm{Hg}$, an analysis of outcomes in the patients achieving this goal, compared with those who did not, demonstrated powerful stroke, mortality, and cardiac benefits of this target. ${ }^{6}$

\section{A DIRECT COMPARISON OF TARGETS}

The action to control cardiovascular risk in diabetes (ACCORD) trial, in some ways designed similarly to the later SPRINT trial, compared outcomes in hypertensive diabetic patients randomized to systolic BPs of $<120$ and $<140 .^{7}$ A major difference between ACCORD and SPRINT 
$\mathrm{HR}(95 \%) \mathrm{Cl}$ ) of $\mathrm{CV}$ events in patients being followed up to 6 years

\begin{tabular}{|c|c|c|}
\hline Fatal and non-fatal cardiac events & & $0.75(0.67-0.83)^{\dagger}$ \\
\hline Fatal and non-fatal stroke & & $0.55(0.46-0.64)^{\dagger}$ \\
\hline All-cause death & & $0.79(0.71-0.88)^{\dagger}$ \\
\hline Myocardial infarction & & $0.86(0.73-1.01)^{\dagger}$ \\
\hline Heart failure hospitalizations & & $0.64(0.55-0.74)$ \\
\hline 0.6 & $\begin{array}{r}0.8 \\
\text { SBP controlled } \\
\text { at } 6 \text { months } \\
(n=10,755)\end{array}$ & $\begin{array}{l}1.2 \\
\text { SBP not controlled } \\
\text { at } 6 \text { months } \\
(n=4,490)\end{array}$ \\
\hline
\end{tabular}

Fig. 1: Effective BP control (SBP $<140 \mathrm{~mm} \mathrm{Hg}$ ) reduces cardiovascular risk (VALUE trial)

was that ACCORD was performed entirely in hypertensive patients with type II diabetes, whereas in SPRINT patients with diabetes were excluded. The primary endpoint in ACCORD was the composite of $\mathrm{CV}$ mortality and nonfatal myocardial infarction (MI) or stroke. This outcome was not significantly different between the two treatment groups, but - of considerable interest - the single outcome of stroke was significantly lower in the intensively treated group.? This finding, in the eyes of many clinicians, was important since stroke is a feared outcome of hypertension, although the failure of other outcomes to benefit from aggressive therapy did raise some questions about aggressive treatment targets in diabetes.

To some extent these concerns were amplified by subsequent analyses of data from other clinical trials. A recent publication based on the avoiding cardiovascular events through combination therapy in patients living with systolic hypertension (ACCOMPLISH) trial included roughly equal proportions of diabetic and nondiabetic patients. ${ }^{8}$ Using the same composite outcome as ACCORD, patients with diabetes in ACCOMPLISH clearly had a significant benefit when their systolic BP was reduced below $140 \mathrm{~mm} \mathrm{Hg}$ when compared with $\geq 140 \mathrm{~mm} \mathrm{Hg}$; further reduction to $<130 \mathrm{~mm} \mathrm{Hg}$ did not provide further benefit, and in patients achieving $<120 \mathrm{~mm} \mathrm{Hg}$ there was a trend to a worsening of this primary endpoint, driven primarily by a significant increase in coronary events. ${ }^{8}$ This finding, together with data from other studies in diabetes, ${ }^{9}$ seems to indicate that there could well be a J-curve phenomenon for systolic BP in treating patients with diabetes. Interestingly, and consistent with the findings from SPRINT, the nondiabetic cohort in ACCOMPLISH appeared to get progressively greater $\mathrm{CV}$ protection with reductions in $\mathrm{BP}$ to around $120 \mathrm{~mm} \mathrm{Hg} .{ }^{8}$ It would be unwise to overinterpret these secondary analyses from ACCOMPLISH, but they help provide a useful perspective in understanding that the differences in results between ACCORD and SPRINT may very well represent true outcomes differences between diabetic and nondiabetic patients when treated aggressively for hypertension.

\section{SPRINT Findings}

As mentioned at the start of this Commentary, SPRINT demonstrated that a more aggressive systolic BP target was superior to a standard target in preventing fatal and nonfatal outcomes in high risk hypertensive patients. This result, however, must be looked at with care. Table 1 gives some of the important underlying data in this trial. For a start, it is critical to understand how BPs were measured. In an attempt to minimize the so-called white coat effect, the SPRINT investigators used a novel method for measuring BP. In brief, patients were seated in a quiet room with the cuff of an automated BP-measuring device attached to the patient's arm. At that point, all medical personnel left the room, and after a 5-minute rest period the device automatically measured three BPs at 1 minute intervals. The average of these three BPs became the official reading. This type of "unobserved" BP measurement has not previously been used in a major outcomes trial, and so this has created difficulty in accurately interpreting the SPRINT BP values in terms of conventional office-measured BPs. There is no question that carefully measured office BPs, even when using good quality automated devices, are higher than the values obtained by the SPRINT method. It is reasonable to assume that this difference is roughly 7 to $10 \mathrm{~mm} \mathrm{Hg}$.

So, as shown in Table 1, the achieved systolic $\mathrm{BP}$ in SPRINT for the intensive treatment group of $121.5 \mathrm{~mm} \mathrm{Hg}$ becomes approximately $129 \mathrm{~mm} \mathrm{Hg}$ by "conventional" office criteria; whereas the SPRINT value of $134.6 \mathrm{~mm} \mathrm{Hg}$ in the standard treatment group should be regarded as approximately $142 \mathrm{~mm} \mathrm{Hg}$ in the office. So, most experts are already re-interpreting the BP results of SPRINT and concluding that a target of around $130 \mathrm{~mm} \mathrm{Hg}$ is probably the desirable goal in high-risk 
Will Recent Trials change the Systolic Treatment Targets?

Table 1: SPRINT: In nondiabetic medium/high-risk hypertensive patients, is there an outcomes benefit in achieving $<120$ vs $<140 \mathrm{~mm} \mathrm{Hg}$ ?

\begin{tabular}{lll}
\hline & $\begin{array}{l}\text { Intensive } \\
\text { treatment }\end{array}$ & $\begin{array}{l}\text { Standard } \\
\text { treatment }\end{array}$ \\
\hline Achieved SPRINT BP* & 121.5 & 134.6 \\
Approximate office BP** & 129 & 142 \\
Mean number of drugs & 3.0 & 1.9 \\
\hline *Mean during treatment period using SPRINT unobserved BP \\
method
\end{tabular}

hypertensive patients. Moreover, there is a concern that treating down to $<120 \mathrm{~mm} \mathrm{Hg}$ by conventional office readings may be taking patients to levels of $\mathrm{BP}$ that in actuality are lower and with a potential for harm than the values reported by SPRINT.

One other important note about SPRINT is also in Table 1. On average, patients in the intensively treated group received one more drug than those in the standard group. As discussed later, this difference in the intensity of drug therapy could have outcomes effects independent of the differences between the two treatment groups in BP.

\section{Interpreting SPRINT}

The detailed results from SPRINT have been widely discussed and I shall not get into much detail here. The primary outcome was a composite of coronary, stroke, heart failure, and CV mortality outcomes. This outcome was reduced by $25 \%$ in the intensively treated group, a powerful and compelling result. The two most impressive components were heart failure, which was reduced by $38 \%$, and CV death, which was reduced by $43 \%$. Total mortality, which was a secondary endpoint, was reduced by a remarkable $27 \%$, a result that in essence put to rest any debate about other individual outcomes in SPRINT. These benefits of intensive treatment were just as compelling in the subgroup of patients aged 75 or more, and appeared to benefit these older patients regardless of their frailty status. ${ }^{10}$

\section{Medication Issue}

Investigators in SPRINT were free to select whichever drugs they preferred in order to help patients achieve their target systolic BPs of $<120$ or $<140 \mathrm{~mm} \mathrm{Hg}$ in this open-label study. Even though patients upon study entry were randomized to either the intensive or standard treatment groups, the investigators were immediately informed of each patient's assignment so that appropriate intensities of treatment could be administered. Table 2 lists some of the major drug types that were used during
Table 2: SPRINT: Drugs that treat or mask heart failure

\begin{tabular}{llll}
\hline & $\begin{array}{l}\text { Intensive } \\
\text { treatment }\end{array}$ & $\begin{array}{l}\text { Standard } \\
\text { treatment }\end{array}$ & Difference \\
\hline Total patients & 4,678 & 4,683 & - \\
Thiazide-like agents & 2,562 & 1,557 & 1,005 \\
RAS blockers & 3,580 & 2,582 & 998 \\
Spironolactone & 405 & 185 & 220 \\
Beta blockers & 1,919 & 1,440 & 479 \\
Heart failure events & 62 & 100 & 38 \\
\hline
\end{tabular}

Question: Can 38 fewer heart failure events be explained by 1,005 more patients on thiazides, 998 on RAS blockers, 220 on spiro, and 479 on BBs - independent of blood pressure effects?

the SPRINT study. ${ }^{1}$ It is quite clear that more drugs were used in the intensive treatment group than in the standard treatment group. Quite remarkably, about 1,000 more patients in the intensive group received diuretics, and a similarly greater number received blockers of the renin angiotensin system angiotensin converting enzyme inhibitors or angiotensin receptor blockers. Even agents like spironolactone and beta blockers were more common. So, as asked in the table, could the difference in heart failure events between the two groups - actually numbering just 38 individual patients - be explained by the far greater use of drugs that, quite apart from their BP-lowering effects, are known to be highly effective in treating the symptoms and improving the prognosis of patients with heart failure? Although not shown in this table, roughly 1,000 more patients in the intensively treated group received calcium channel blockers which, together with the drug classes already mentioned, could also have contributed to the mortality benefits observed with intensive treatment. These questions deserve to be carefully considered because while there is no question that the intensive treatment protocol in SPRINT had powerful CV and mortality benefits, it may be important in future analyses of the SPRINT database, or in further trials designed to explore these issues, to learn more about optimal drug combinations that - beyond BP reductioncan optimize the benefits of hypertension therapy.

\section{A Need for Caution}

Two recent clinical trials provide useful perspectives on the SPRINT data and serve to warn us that being excessively aggressive in treating hypertension may create important problems for patients. One important trial was CLARIFY, a carefully conducted registry study of major outcomes in hypertensive patients with stable coronary disease. ${ }^{11}$ Like some of the studies already discussed, CLARIFY had a primary outcome of CV mortality, or nonfatal MI, or stroke. When looking at $10 \mathrm{~mm} \mathrm{Hg}$ systolic BP intervals, the CLARIFY investigators found that the $10 \mathrm{~mm} \mathrm{Hg}$ range below $130 \mathrm{~mm} \mathrm{Hg}$ was associated with the lowest event rate. Compared with that reference 
group, patients with BPs between 140 and $149 \mathrm{~mm} \mathrm{Hg}$ were about $50 \%$ more likely to have the primary endpoint, and those $\geq 150 \mathrm{~mm} \mathrm{Hg}$ were almost 2.5-fold more likely to have events. However, of particular importance in considering the SPRINT data, CLARIFY demonstrated that achieved office systolic BPs $<120 \mathrm{~mm} \mathrm{Hg}$ were associated with a $56 \%$ greater event rate than at the $<130 \mathrm{~mm} \mathrm{Hg}$ target. Since these are high-risk patients similar to those in SPRINT, it does seem important that our interpretation of the SPRINT BPs - in particular, that the intensively treated SPRINT group should be regarded as having achieved $130 \mathrm{~mm} \mathrm{Hg}$ rather than $120 \mathrm{~mm} \mathrm{Hg}$ - is of great importance. Clinicians who interpret the SPRINT data in a literal way, and aim to achieve office BPs $<120 \mathrm{~mm} \mathrm{Hg}$ in their high-risk patients, could be at risk of causing harm.

Another major trial that was announced soon after SPRINT was the heart outcomes prevention experience-3 (HOPE-3) that was performed in a large community cohort with patients at relatively low CV risk. ${ }^{12}$ In fact, HOPE-3 excluded patients who had histories of prior $\mathrm{CV}$ events, although they did include people with more conventional CV risk factors. The HOPE-3 trial failed to find an overall benefit of active treatment (an ARB plus a thiazide) compared with placebo. However, in a prespecified analysis, patients in the highest tertile for baseline BPs (roughly $>140 \mathrm{~mm} \mathrm{Hg}$ ) did have a significant 24\% reduction in the composite $\mathrm{CV}$ outcome; patients in the middle tertile (roughly 130-140 mm Hg) had a neutral outcome with similar event rates in the actively treated and placebo groups. Of concern, patients in the lowest tertile (roughly $\leq 130 \mathrm{~mm} \mathrm{Hg}$ ) showed a trend to increased $\mathrm{CV}$ events in the actively treated group compared with placebo. ${ }^{12}$ This finding also provides a warning to clinicians that over-interpreting SPRINT and treating patients in the range 120 to $130 \mathrm{~mm} \mathrm{Hg}$ to achieve office values $<120 \mathrm{~mm} \mathrm{Hg}$ could possibly precipitate, rather than prevent, major events in lower-risk patients.

\section{RECOMMENDED SYSTOLIC BP TARGETS}

A summary of evidence-based systolic BP targets is shown in Table 3. Based on the SPRINT trial, it appears most appropriate to recommend an office target of

Table 3: Office systolic BP targets

\footnotetext{
- For nondiabetic adult patients at medium to high CV risk, target should be $<130 \mathrm{~mm} \mathrm{Hg}^{6,10,13,14}$

- For diabetic patients the target should be $<140 \mathrm{~mm} \mathrm{Hg}$ (OK if close to $130 \mathrm{~mm} \mathrm{Hg})^{7-9}$

- For patients at low/medium CV risk, the target is $<140 \mathrm{~mm} \mathrm{Hg}^{12}$

- Could be harm with office SBP target of $<120 \mathrm{~mm} \mathrm{Hg}^{11,12}$

Note: Data are strongest in patients aged $>55$. Lower BP targets may be appropriate in younger adults, but evidence is lacking
}

$130 \mathrm{~mm} \mathrm{Hg}$ in nondiabetic hypertensive patients at medium-to-high CV risk. This recommendation is supported by other reports as well. ${ }^{13,14}$ For patients with diabetes, ACCORD and other trials ${ }^{7-9}$ indicate that a target of $<140 \mathrm{~mm} \mathrm{Hg}$ is justified; perhaps in these patients, it is reasonable to approach $130 \mathrm{~mm} \mathrm{Hg}$, although not below. For patients at low-to-medium CV risk, the target should be $<140 \mathrm{~mm} \mathrm{Hg}$, particularly in view of the recent HOPE-3 trial. $^{12}$

It should be emphasized that there has not been a systematic randomized trial that has prospectively tested office systolic BPs of $<120 \mathrm{~mm} \mathrm{Hg}$ (remember: The target of $<120 \mathrm{~mm} \mathrm{Hg}$ in SPRINT utilized a unique BP measurement method that corresponds more closely to an office value of around $130 \mathrm{~mm} \mathrm{Hg}$ ). Indeed, as discussed earlier, achieving office BPs of $<120 \mathrm{~mm} \mathrm{Hg}$ have the potential to cause harm. ${ }^{11,12}$

In a way, these recommendations could be simplified by suggesting a general target of $130 \mathrm{~mm} \mathrm{Hg}$, or slightly above, which should satisfy the needs of almost all hypertensive patients. It is also worth adding that, as SPRINT so capably demonstrated, elderly patients have done very well when treated to office values of around $130 \mathrm{~mm} \mathrm{Hg}$, even those regarded as "frail." One major omission from these recommendations is for younger patients. Almost every clinical trial cited to support BP target recommendations is based on patients aged 55 or older, with an overall average age in these trials in the mid to high 60s. It is probably appropriate to target younger patients in their 20s, 30s, and 40s to $130 \mathrm{~mm} \mathrm{Hg}$, although if the pressure falls closer to $120 \mathrm{~mm} \mathrm{Hg}$ without the need for intensive pharmacologic intervention, that level of BP is probably very acceptable.

\section{GLOBAL ISSUES}

The recommendations made so far in these comments have been based on communities with reasonable access to health care. But as shown in Table 4, in many low- or middle-income countries resources are severely limited. There is often a shortage of trained health workers, including those with the ability to perform BP measurements. Another problem is that the cost of acquiring and maintaining simple BP measuring devices is often

Table 4: Global issues: Limited resources

\footnotetext{
- Shortage of trained health workers

- Cost of acquiring and maintaining BP measurement devices

- Identifying key risk factors not routinely available, e.g., renal function/glucose/lipids/X-rays/ECGs often limited to patients with manifest disease findings

- Limited logistical support for training, record-keeping, and scheduling

- Access to drugs can be highly variable
} 
prohibitive. In addition, it becomes very difficult to measure or estimate overall CV risk in patients in such communities because many of the standard evaluations, including routine blood tests, $\mathrm{X}$-rays, and electrocardiograms (ECGs) are simply not commonly available. On top of that, administrative logistics are limited, making it difficult to perform essential tasks like record keeping and patient scheduling.

And finally, it should be emphasized that access to drugs is highly variable. Many such communities depend on donations of drug supplies, which often means that there is inconsistency from one patient visit to the next in the type of therapy available to manage the patient's hypertension.

\section{STRATEGIES IN GLOBAL GUIDELINES}

It is probably most reasonable in low-resource settings to reserve treatment for patients who are at greater risk, which often means those patients with untreated BPs at higher levels are given greater priority. The recommendation of $130 \mathrm{~mm} \mathrm{Hg}$ that was discussed previously may be too ambitious in many areas, so $140 \mathrm{~mm} \mathrm{Hg}$ should probably be a more universal target. Another factor that differentiates the care of patients in areas with limited resource is the types of lifestyle therapies that can be recommended. For instance, recommending weight loss is not practical in low-income areas where most patients have a body mass index that is clearly in the normal range by Western standards. Similarly, in hot and tropical climates, it is hard to manage sodium intake, particularly in patients engaged in manual work who may experience a great deal of sodium loss during the day.

Based on some of these considerations, the guidelines listed in Table 5 seem reasonable for communities with limited resources. Very simply, we should use a systolic $\mathrm{BP}$ of $140 \mathrm{~mm} \mathrm{Hg}$ as the threshold and target for treatment, and at times it may be economically necessary to delay therapy at levels even higher than that. Since traditional office BP measurement techniques are difficult to teach, it is definitely preferable to use automated

Table 5: Global hypertension guidelines for low-resource settings

\footnotetext{
- Since cannot usually stratify by risk, use systolic BP of $140 \mathrm{~mm} \mathrm{Hg}$ as threshold and target

- Traditional BP measurements difficult to teach, so use automated devices with simplified protocol

- Lifestyle advice tailored to environment

- For "one drug fits all" settings, a CCB may be the overall best choice (works in most ethnicities and ages)

- For more severe hypertension, 2 or 3 drug combinations (single pill, if available) based on RAS/CCB/diuretic in hope of replicating powerful benefits of SPRINT "Intensive Treatment"
}

devices that do not require operator training. We must recognize that the cost of these devices, and even the cost of the batteries to operate these devices, can be a major consideration, but this is the most accurate and consistent way to measure BP.

Lifestyle modifications must be based on the environment, and in many cases are difficult to enforce. The one exception may be within those cultures where very high salt intake is part of the standard diet, and where a community or country-wide reduction in food salt content might produce a broad-based benefit.

In settings where it is not possible to get access to multiple drug classes, it might be most desirable to select a dihydropyridine calcium channel blocker as the overall best choice since these agents appear to work well regardless of patient ethnicity and age. For more sever hypertension, it would be highly advantageous to obtain single-pill combinations that offer two or even three-drug options based on the three-core hypertension therapies: Blockers of the renin angiotensin system/ calcium channel blockers/thiazide diuretics. It should be emphasized that this relatively simple strategy was the one used most commonly in the highly successful SPRINT trial. Many of these combination products are now generically available, although their cost may still be daunting in many parts of the world. Even so, since SPRINT the incentive to upgrade hypertension care has become stronger than ever.

\section{REFERENCES}

1. The SPRINT Research Group, Wright JT Jr, Williamson JD, Whelton PK, Snyder JK, Sink KM, Rocco MV, Reboussin DM, Rahman M, Oparil S, et al. A randomized trial vs. standard blood pressure control. N Engl J Med 2015 Nov 26;373(22): 2103-2116.

2. Chobanian AV, Bakris GL, Black HR, Cushman WC, Green LA, Izzo JL Jr, Jones DW, Materson BJ, Oparil S, Wright JT Jr, et al. The Seventh Report of the Joint National Committee on Prevention, Detection, Evaluation, and Treatment of High Blood Pressure: the JNC7 report. JAMA 2003 May 21;289(19): 2560-2572.

3. Mancia G, Fagard R, Narkiewicz K, Redón J, Zanchetti A, Böhm M, Christiaens T, Cifkova R, De Backer G, Dominiczak A, et al. 2013 ESH/ESC guidelines for the management of arterial hypertension. J Hypertens 2013 Jul;31(7):1281-1357.

4. SHEP Cooperative Research Group. Prevention of stroke by antihypertensive drug treatment in older persons with isolated systolic hypertension. Final results of the Systolic Hypertension in the Elderly Program (SHEP). JAMA 1991 Jun 26;265(24):3255-3264.

5. James PA, Oparil, S, Carter BL, Cushman WC, DennisonHimmelfarb C, Handler J, Lackland DT, LeFevre ML, MacKenzie TD, Ogedegbe O, et al. Evidence based guidelines for the management of high blood pressure in adults: report from the panel members appointed to the Eighth Joint National Committee (JNC8). JAMA 2014 Feb 5;311(5):507-520. 
6. Weber MA, Julius S, Kjeldsen SE, Brunner HR, Ekman S, Hansson L, Hua T, Laragh JH, McInnes GT, Mitchell L, et al. Blood pressure dependent and independent effects of antihypertensive treatment on clinical events in the VALUE trial. Lancet 2004 Jun 19;363(9426):2049-2051.

7. ACCORD Study Group, Cushman WC, Evans GW, Byington RP, Goff DC Jr, Grimm RH Jr, Cutler JA, SimonsMorton DG, Basile JN, Corson MA, et al. Effects of intensive blood pressure control in type 2 diabetes mellitus. N Engl J Med 2010 Apr 29;362(17):1575-1585.

8. Weber MA, Bloch M, Bakris GL, Weir MR, Zappe DH, Dahlof B, Velazquez EJ, Pitt B, Basile JN, Jamerson K, et al. Cardiovascular outcomes according to systolic blood pressure in patients with and without diabetes: an ACCOMPLISH substudy. J Clin Hypertens 2016 Apr;18(4):299-307.

9. Brunström M, Carlberg B. Effect of antihypertensive treatment at different blood pressure levels in patients with diabetes mellitus: systematic review and meta-analyses. BMJ 2016 Feb 24;352:i717.

10. Williamson JD, Supiano MA, Applegate WB, Berlowitz DR, Campbell RC, Chertow GM, Fine LJ, Haley WE, Hawfield AT, $\mathrm{Ix} \mathrm{JH}$, et al. Intensive vs standard blood pressure control and cardiovascular disease outcomes in adults aged $\geq 75$ years: a randomized clinical trial. JAMA 2016 Jun 28;315(24): 2673-2682.

11. Vidal-Petiot E, Ford I, Greenlaw N, Ferrari R, Fox KM, Tardif JC, Tendera M, Tavazzi L, Bhatt DL, Steg PG, et al. Cardiovascular event rates and mortality according to achieved systolic and diastolic blood pressure in patients with stable coronary artery disease: an international cohort study. Lancet 2016 Oct;388(10056):2142-2152.

12. Lonn EM, Bosch J, López-Jaramillo P, Zhu J, Liu L, Pais P, Diaz R, Xavier D, Sliwa K, Dans A, et al. Blood pressure lowering in intermediate risk persons without cardiovascular disease. N Engl J Med 2016 May 26;374(21):2009-2020.

13. Weber MA, Bakris GL, Hester A, Weir MR, Hua TA, Zappe D, Dahlof B, Velazquez EJ, Pitt B, Jamerson K. Systolic blood pressure and cardiovascular outcomes during treatment of hypertension. Am J Med 2013 Jun;126(6):501-508.

14. Ettehad D, Emdin CA, Kiran A, Anderson SG, Callender T, Emberson J, Chalmers J, Rodgers A, Rahimi K. Blood pressure lowering for prevention of cardiovascular disease and death: a systematic review and meta-analysis. Lancet 2016 Mar 5;387(10022):957-967. 